\title{
ASPECTOS QUE PODEM INFLUENCIAR A IMPLEMENTAÇÃO DA MANUFATURA ENXUTA EM ORGANIZAÇÕES
}

\section{José Salvador da Motta Reis ${ }^{1}$, Weslei Jardim Batista ${ }^{1}$, Maximilian Espuny ${ }^{1}$, Fernanda De Oliveira Silva ${ }^{2}$, Jorge Muniz Junior ${ }^{1}$ e Otávio José de Oliveira'.}

\author{
${ }^{1}$ Universidade Estadual Paulista /Faculdade de Engenharia de Guaratinguetá, Avenida Dr. Ariberto \\ Pereira da Cunha, 333, Bairro - 12244-000 - Guaratinguetá-SP, Brasil, jmottareis@gmail.com, \\ wesleibatista.adm@gmail.com, maximilian.espuny@unesp.br, jorge.muniz@unesp.br e \\ otavio.oliveira@unesp.br. \\ ${ }^{2}$ Universidade Federal do ABC, Alameda da Universidade, s/no, Anchieta - 09606-045 - São Bernardo \\ do Campo - SP, Brasil, fernanda.o@aluno.ufabc.edu.br.
}

Resumo - Esse estudo teve por objetivo identificar e descrever, por meio de uma pesquisa bibliográfica, os aspectos que influenciam a implementação da Manufatura Enxuta em organizações. Para que esse objetivo fosse alcançado, foi realizada uma pesquisa aplicada, de natureza exploratória com abordagem qualitativa, sendo que foram adotados como método e procedimentos técnicos, respectivamente, a pesquisa bibliográfica e a revisão de literatura. Ressalta-se que os dados utilizados foram coletados na base SCOPUS no mês de maio de 2019. Como principais resultados, pode-se apontar que foram identificados que os fatores que influenciam a implementação da Manufatura Enxuta são: a cultura organizacional, treinamento, recursos, gerência, comprometimento e a compreensão da ME. Com relação à esses fatores, destaca-se o forte impacto do alinhamento com a gerência, que pode ser considerado um ponto-chave ao obter sucesso ou não no projeto, além do comprometimento com o projeto e a dificuldade em compreender a Manufatura Enxuta.

Palavras-chave: Lean Manufacturing, Influência, Produção Enxuta, Implementação. Área do Conhecimento: Engenharias.

\section{Introdução}

O sistema de produção da Toyota é a base da Manufatura Enxuta (ME), sendo que o Instituto de Tecnologia do Massachusetts (MIT) explanou ao mundo nos anos de 1990 a mesma para redefinir o sistema de produção da Toyota. Destaca-se que a filosofia Enxuta iniciou-se como uma operação profissional no setor automobilístico na década de 1980 (TIWARI; TIWARI, 2018). O precursor da ideia de produção enxuta foi o engenheiro-chefe Taiichi Ohno, da Montadora Toyota de veículos. A ME pode ser considerada eficiente porque usa menos: produção em massa, esforço humano na fábrica, espaço de produção, investimento em ferramentas; e trabalho de engenharia para desenvolver um novo produto (WIRKUS; CHMIELARZ; CHMIELARZ, 2018).

O levantamento da literatura evidencia diversos tipos de barreiras que influenciam na implementação da Manufatura Enxuta em organizações. Pesquisadores investigaram e analisaram as influências críticas na implementação da Manufatura Enxuta e identificaram que o sucesso da implementação não está apenas na aplicação de ferramentas e técnicas apropriadas, mas também na participação e liderança da alta gerência, atitude dos funcionários, recursos e cultura organizacional (SHRIMALI; SONI; PAWAR, 2018). Nesse contexto, surge a seguinte problemática: quais são os aspectos que podem influenciar a implementação dessa filosofia nas organizações? Respondendo a esse questionamento, o objetivo dessa pesquisa é identificar e descrever os aspectos que influenciam a implementação da Manufatura Enxuta em organizações, por meio de pesquisa bibliográfica. Destaca-se que a literatura existente na academia a respeito da Manufatura Enxuta é densa e aborda os diferentes aspectos sobre esta temática. Este fato reflete a relevância que a questão representa para a comunidade acadêmica e o ambiente industrial (SATOLO et al., 2017).

Esse trabalho encontra-se organizado em cinco seções, incluindo essa Introdução. Na segunda seção, serão apresentados conceitos referentes à temática em pauta que servirão de arcabouço teórico nesse estudo. Na terceira seção, serão apresentados os procedimentos metodológicos 
adotados durante toda a realização desse trabalho de pesquisa. Na quarta seção, serão apresentados e discutidos os resultados obtidos por meio da coleta de dados e da revisão de literatura e, finalizando esse estudo, serão apresentadas as considerações finais e principais.

\section{Metodologia}

Apesar de ser nomeada como "Manufatura Enxuta" (ME), a aplicação dessa filosofia excede o campo da produção, sendo algo mais complexo como um sistema inter-relacionado de práticas e crenças e valores. A ME, devido a sua complexidade, cria opiniões divergentes gerando confusões no nível teórico e prático durante a sua implementação (TAHERIMASHHADI; RIBAS, 2018). São trabalhados no Sistema Toyota de Produção (TPS) os seguintes princípios: valor, fluxo de valor, fluxo, fluxo puxado e perfeição. Ressalta-se que o princípio de respeito pelas pessoas é considerado pelos fundadores do TPS como base fundamental na cooperação e confiança com todos os funcionários (BAUER et al., 2018). A ideia (ou método) é aceita ou rejeitada por indivíduos de diferentes culturas relacionados ao processo demonstrando a influência do fator cultura organizacional como chave para implementar a ME. Destaca-se que o desafio de implementar e sustentar os processos enxutos reside na necessidade de identificar a cultura organizacional do ambiente (PAKDIL; LEONARD, 2015).

O alinhamento da liderança é o começo para a transformação assim como as normas sociais, características dos indivíduos envolvidos, a forma de comunicação e o treinamento. $O$ suporte gerencial impacta a implementação da ME de forma negativa e positiva. Ressalta-se que a falta de apoio e alinhamento da liderança pode levar a uma série de questões como: acesso limitado a recursos, longos processos de tomada de decisão e falhas de comunicação (TAHERIMASHHADI; RIBAS, 2018). A Manufatura Enxuta é fundamentada por um sistema de produção do tipo puxada, na produção de apenas itens necessários, no tempo necessário, nas quantidades necessárias, reduzindo os desperdícios e assegurando a qualidade do produto (JAYARAM; VICKERY; DROGE, 2008). Destaca-se que os desperdícios são separados em sete categorias, sendo elas: estoque desnecessário, excesso de produção, espera, excesso de transporte, processamento inadequado, defeitos e movimentações desnecessárias (BAYSAN et al., 2019) aumentando, dessa forma, o valor das atividades envolvidas na produção a fim de alcançar um fluxo consistente e limpo de produção (SHRIMALI; SONI; PAWAR, 2018), contribuindo para aprimorar a eficiência e melhorando a capacidade em responder as eventuais mudanças do processo tão rápido quanto exigido pelo cliente (ALKHORAIF; MCLAUGHLIN, 2018).

A pesquisa pode ser classificada como uma pesquisa aplicada, de natureza exploratória com abordagem qualitativa. Como método e procedimentos técnicos foram adotados, respectivamente, a pesquisa bibliográfica e a revisão de literatura com o intuito de identificar aspectos que influenciam a Manufatura Enxuta. Destaca-se que os dados utilizados no estudo foram coletados no mês de maio de 2019 na base de dados SCOPUS e que na busca foram utilizadas como descritores as palavras "Aspects", "Influence" e "Lean Manufacturing". Os trabalhos utilizados nessa pesquisa foram hierarquizados de acordo com o índice de citações por título. Os dados foram tratados por meio do software Microsoft Excel ${ }^{\circledR}$ e os resultados obtidos serão apresentados e discutidos na próxima seção

\section{Resultados}

Identificou-se na pesquisa objetivando aspectos que influenciam a Manufatura Enxuta como pode ser conferido na Tabela 1.

Tabela 1- Autores mais citados entre 2009 e 2019.

\begin{tabular}{cc}
\hline Descritores & Número de trabalhos indexados \\
\hline "Aspects", "Influence" e "Lean Manufacturing" & 15 \\
"Organizational Culture" e "Lean Manufacturing" & 34 \\
"Training" e "Lean Manufacturing" & 273 \\
"Resources" e "Lean Manufacturing" & 575 \\
"Management" e "Lean Manufacturing & 1656
\end{tabular}

XXIII Encontro Latino Americano de Iniciação Científica, XIX Encontro Latino Americano de Pós-Graduação e 2 IX Encontro de Iniciação à Docência - Universidade do Vale do Paraíba. 
"Commitment" e Lean Manufacturing"

90

"Understanding" e "Lean Manufacturing

Fonte: Próprios autores.

Os trabalhos indexados na busca da Base SCOPUS com as palavras chaves "Aspects", "Influence" e "Lean Manufacturing" são apresentados na Tabela 2, sendo hierarquizados de acordo com o índice de citações por título.

Tabela 2- Artigos mais citados na pesquisa bibliográfica de fatores que influenciam a ME.

\begin{tabular}{|c|c|c|}
\hline Autores & Título & Fonte \\
\hline $\begin{array}{l}\text { Jayaram et al. } \\
\qquad(2008)\end{array}$ & $\begin{array}{l}\text { Relationship building, lean strategy and firm performance: na } \\
\text { exploratory study in the automotive supplier industry }\end{array}$ & $\begin{array}{l}\text { International Journal of Production } \\
\text { Research }\end{array}$ \\
\hline $\begin{array}{l}\text { Pakdil et al. } \\
\qquad(2015)\end{array}$ & $\begin{array}{l}\text { The effect of organizational culture on implementing and } \\
\text { sustaining lean processes }\end{array}$ & $\begin{array}{l}\text { Journal of Manufacturing Technology } \\
\text { Management }\end{array}$ \\
\hline $\begin{array}{l}\text { Murugesan et } \\
\text { al.(2012) }\end{array}$ & $\begin{array}{l}\text { Competitive advantage of World Class Manufacturing System } \\
\text { (WCMS) - a study of manufacturing companies in South India }\end{array}$ & European Journal of Social Sciences \\
\hline $\begin{array}{l}\text { Cantamessa et } \\
\text { al. (2009) }\end{array}$ & $\begin{array}{l}\text { Beyond lean manufacturing: Developing an integrated } \\
\text { methodology to design effective manufacturing systems }\end{array}$ & $\begin{array}{c}2008 \text { Proceedings of the 9th Biennial } \\
\text { Conference on Engineering Systems } \\
\text { Design and Analysis }\end{array}$ \\
\hline $\begin{array}{l}\text { Satolo et al. } \\
\qquad(2017)\end{array}$ & $\begin{array}{l}\text { Lean production in agribusiness organizations: multiple case } \\
\text { studies in a developing country }\end{array}$ & International Journal of Lean Six Sigma \\
\hline $\begin{array}{l}\text { Shaikh et al. } \\
\qquad(2012)\end{array}$ & $\begin{array}{l}\text { Investigating the effects of physical and cognitive demands on } \\
\text { the quality of performance under different pacing levels }\end{array}$ & Work \\
\hline $\begin{array}{l}\text { Tortorella et al. } \\
\qquad(2017)\end{array}$ & $\begin{array}{l}\text { Lean manufacturing implementation, context and behaviors of } \\
\text { multi-level leadership A mixed-methods exploratory research }\end{array}$ & $\begin{array}{l}\text { Journal of Manufacturing Technology } \\
\text { Management }\end{array}$ \\
\hline $\begin{array}{l}\text { Tortorella et al. } \\
\qquad(2019)\end{array}$ & $\begin{array}{l}\text { The moderating role of just-in-time on sociotechnical practices' } \\
\text { effect over quality and workers' health }\end{array}$ & $\begin{array}{l}\text { Human Factors and Ergonomics In } \\
\text { Manufacturing }\end{array}$ \\
\hline $\begin{array}{l}\text { Taherimashhadi } \\
\text { et al. (2018) }\end{array}$ & A Model to align the organizational culture to Lean & $\begin{array}{l}\text { Journal of Industrial Engineering and } \\
\text { Management }\end{array}$ \\
\hline $\begin{array}{l}\text { Tortorella et al. } \\
\qquad(2017)\end{array}$ & $\begin{array}{l}\text { The adoption of socio-technical and JIT practices and its } \\
\text { relationship with the performance of quality and workers' health }\end{array}$ & $\begin{array}{c}\text { Proceedings of the International } \\
\text { Conference on Industrial Engineering } \\
\text { and Operations Management }\end{array}$ \\
\hline
\end{tabular}

Fonte: Próprios autores.

A partir da análise da Tabela 1, pode-se constatar que os fatores de influência na implementação da ME mais citados por autores nos trabalhos encontrados na busca foram: Cultura Organizacional, Treinamento, Recursos, Gerência, Comprometimento e Compreensão da Manufatura Enxuta. No Tabela 2, pode-se observar os fatores de influência e os autores que os apontaram em seus respectivos trabalhos de pesquisa.

\section{Discussão}

A partir da análise do Quadro 1, pode-se constatar que os fatores de influência na implementação da ME mais citados por autores nos trabalhos encontrados na busca foram: Cultura Organizacional, Treinamento, Recursos, Gerência, Comprometimento e Compreensão da Manufatura Enxuta. No Quadro 2, pode-se observar os fatores de influência e os autores que os apontaram em seus respectivos trabalhos de pesquisa.

Cada organização tem uma cultura própria proveniente dos funcionários e local onde reside e isso impacta na implementação de qualquer projeto empresarial. Segundo Pakdil e Leonard (2015) as organizações recebem uma influência da cultura organizacional afetando processos de Manufatura Enxuta que podem impedir o sucesso na implementação de programas de melhoria. 
O processo como um todo de uma empresa é impactado pela falta de treinamento, mesmo que de apenas um colaborador, pois uma engrenagem que não funciona atrapalha ou para todo um processo maior assim como a implementação de qualquer ferramenta ou método. Nesse contexto, Netland (2016) aponta que a deficiência do treinamento é evidenciada quando o funcionário está limitado devido ao seu conhecimento a respeito de sua função, impossibilitando que atinja os objetivos e impactando no processo da ME.

$\mathrm{Na}$ falta dos mínimos recursos a implementação de qualquer método se faz negligenciada, sendo impossibilitado o prosseguimento de implementação da Manufatura Enxuta. Segundo Shrimali et al. (2018), na falta de recursos a implementação de qualquer método se faz negligenciada, sendo impossibilitado o prosseguimento de implementação da Manufatura Enxuta.

O alinhamento e suporte gerencial impacta a implementação da Manufatura Enxuta de forma negativa e positiva. Destaca-se que a falta de compromisso e apoio da liderança possibilita uma série questões negativas dificuldade de acesso a recursos por funcionários, tomadas de decisões demoradas e falhas de comunicação (TAHERIMASHHADI; RIBAS, 2018).

A resistência existe em diferentes níveis gerenciais de uma organização como: estratégico, administrativo e operacional (JADHAV; MANTHA; RANE, 2014), evidenciando que as práticas de qualquer empresa devem vir de cima para baixo, ou seja: os líderes apresentam visões, valores e métodos diferentes e orientam o sucesso individual e coletivo para que suas equipes sejam bemsucedidas. Nesse contexto, Tortorella et al. (2017) apontam que as organizações com níveis mais altos de implementação devem observar os gerentes e diretores como fatores de influência na Manufatura Enxuta pois são responsáveis por um alto número de seguidores dentro da organização.

É necessário que o corpo de colaboradores esteja motivado e alinhado com a visão e filosofia de ME dá empresa, pois são as pessoas que impactarão diretamente o produto ou serviço acabado. Segundo Shrimali et al. (2018), o comprometimento e proatividade da mão de obra interfere incisivamente para obter os resultados desejados na implementação da ME.

A dificuldade em compreender a Manufatura Enxuta existe em virtude das diferentes definições e caracterizações divergem entre si a respeito do que consiste a fabricação Enxuta, conflitando no entendimento da filosofia (ABOLHASSANI; LAYFIELD; GOPALAKRISHNAN, 2016). Destaca-se que essas divergências criam incertezas no momento de implementar a ME. Segundo Fagerlind et al. (2015), a compreensão da filosofia da ME é um fator influente na implementação da ME em virtude da dificuldade em conceituar de forma clara e definitiva a filosofia da Manufatura Enxuta.

\section{Conclusão}

Essa investigação teve por objetivo identificar e descrever, por meio de uma pesquisa bibliográfica, os aspectos que influenciam a implementação da Manufatura Enxuta em organizações. Para que esse objetivo fosse alcançado, foi realizada uma pesquisa aplicada, de natureza exploratória com abordagem qualitativa, sendo que foram adotados como método e procedimentos técnicos, respectivamente, a pesquisa bibliográfica e a revisão de literatura. Ressalta-se que os dados utilizados foram coletados na base SCOPUS no mês de maio de 2019. Como principais resultados, pode-se apontar que foram identificados que os fatores que influenciam a implementação da Manufatura Enxuta são: a cultura organizacional, treinamento, recursos, gerência, comprometimento e a compreensão da ME. Com relação à esses fatores, destaca-se o forte impacto do alinhamento com a gerência, que pode ser considerado um ponto-chave ao obter sucesso ou não no projeto, além do comprometimento com o projeto e a dificuldade em compreender a Manufatura Enxuta.

Como limitação, aponta-se o pequeno número de trabalhos indexados na base SCOPUS que abordavam a temática em pauta o que, de certa forma, permitiu uma visão limitada do tema investigado. Sugere-se, portanto, que o método utilizado nessa investigação seja replicado abrangendo outras bases de dados como, por exemplo, a Web of Science (WoS), a Emerald Insight, Science Direct, Google Acadêmico, SciELO, dentre outras. Dessa forma, os dados obtidos poderão ser comparados e possibilitarão uma visão mais abrangente sobre os fatores de implementação da ME no âmbito organizacional. Destaca-se que a referida pesquisa não esgota o assunto e que novos estudos sobre essa temática estão sendo realizados.

\section{Agradecimentos}

XXIII Encontro Latino Americano de Iniciação Científica, XIX Encontro Latino Americano de Pós-Graduação e 4 IX Encontro de Iniciação à Docência - Universidade do Vale do Paraíba. 
Este trabalho foi viabilizado com o apoio do CNPq PQ Proc. 312894/2017-1.

\section{Referências}

ABOLHASSANI, A.; LAYFIELD, K.; GOPALAKRISHNAN, B. Lean and US manufacturing industry: popularity of practices and implementation barriers. International Journal of Productivity and Performance Management, v. 65, n. 7, p. 875-897, 12 set. 2016.

ALKHORAIF, A.; MCLAUGHLIN, P. Lean implementation within manufacturing SMEs in Saudi Arabia: Organizational culture aspects. Journal of King Saud University - Engineering Sciences, v. 30, n. 3, p. 232-242, jul. 2018.

BAUER, $\mathrm{H}$. et al. Integration of Industrie 4.0 in Lean Manufacturing Learning Factories. Procedia Manufacturing, v. 23, n. 2017, p. 147-152, 2018.

BAYSAN, S. et al. A simulation-based methodology for the analysis of the effect of lean tools on energy efficiency: An application in power distribution industry. Journal of Cleaner Production, v. 211, p. 895-908, fev. 2019.

FAGERLIND STÅHL, A.-C. et al. Lean production tools and decision latitude enable conditions for innovative learning in organizations: A multilevel analysis. Applied Ergonomics, v. 47, n. 47, p. 285291, mar. 2015.

JADHAV, J.; MANTHA, S.; RANE, S. Exploring barriers in lean implementation. International Journal of Lean Six Sigma, v. 5, n. 2, p. 122-148, 27 maio 2014.

JAYARAM, J.; VICKERY, S.; DROGE, C. Relationship building, lean strategy and firm performance: na exploratory study in the automotive supplier industry. International Journal of Production Research, v. 46, n. 20, p. 5633-5649, 15 out. 2008.

NETLAND, T. H. Critical success factors for implementing lean production: the effect of contingencies. International Journal of Production Research, v. 54, n. 8, p. 2433-2448, 17 abr. 2016.

PAKDIL, F.; LEONARD, K. M. The effect of organizational culture on implementing and sustaining lean processes. Journal of Manufacturing Technology Management, v. 26, n. 5, p. 725-743, jun. 2015.

SATOLO, E. G. et al. Lean production in agribusiness organizations: multiple case studies in a developing country. International Journal of Lean Six Sigma, v. 8, n. 3, p. 335-358, 7 ago. 2017.

SHRIMALI, A. K.; SONI, V. K.; PAWAR, S. S. Interpretive Structural Modeling of identified Barriers to Lean Implementation in SMEs. MATEC Web of Conferences, v. 183, p. 01008, 2018.

TAHERIMASHHADI, M.; RIBAS, I. A Model to align the organizational culture to Lean. Journal of Industrial Engineering and Management, v. 11, n. 2, p. 207, 6 abr. 2018.

TIWARI, R. K.; TIWARI, J. K. Evaluating Lean Performance of Indian Small and Medium Sized Enterprises in Automotive Sector. Open Engineering, v. 8, n. 1, p. 293-306, 26 set. 2018.

TORTORELLA, G. et al. Lean manufacturing implementation, context and behaviors of multi-level leadership: A mixed-methods exploratory research. Journal of Manufacturing Technology 
Management, v. 28, n. 7, p. 867-891, 4 set. 2017.

WIRKUS, M.; CHMIELARZ, A.; CHMIELARZ, A. Integration of Lean Management With Iso Management Systems in Enterprise. Management and Production Engineering Review, v. 9, n. 3, p. 100-107, 2018. 